\title{
1 Aberrant accumulation of NIK promotes tumorigenicity by 2 dysregulating post-translational modifications in breast cancer
}

4 Yusuke Hayashi ${ }^{1,2}$, Jun Nakayama ${ }^{1,2^{*}}$, Mizuki Yamamoto $^{3}$, Masashi Maekawa ${ }^{4,5}$, Shinya

$5 \quad$ Watanabe $^{6}$, Shigeki Higashiyama ${ }^{4,5,7}$, Jun-ichiro Inoue ${ }^{8}$, Yusuke Yamamoto ${ }^{2}$, Kentaro Semba $^{1,6^{*}}$

6

7 1. Department of Life Science and Medical Bioscience, School of Advanced Science and

8 Engineering, Waseda University, 2-2 Wakamatsu-cho, Shinjuku-ku, Tokyo, 162-8480, Japan.

9 2. Laboratory of Integrative Oncology, National Cancer Center Research Institute, 5-1-1 Tsukiji,

10 Chuo-ku, Tokyo, 104-0045, Japan.

11 3. Research Center for Asian Infectious Diseases, The Institute of Medical Science, The

12 University of Tokyo, Shirokane-dai, Minato-ku, Tokyo 108-8639, Japan.

13 4. Division of Cell Growth and Tumor Regulation, Proteo-Science Center, Ehime University,

14 Toon, 791-0295, Japan.

15 5. Department of Biochemistry and Molecular Genetics, Ehime University Graduate School of

16 Medicine, Toon, 791-0295, Japan.

17 6. Translational Research Center, Fukushima Medical University, Fukushima, 960-1295, Japan.

18 7. Department of Molecular and Cellular Biology, Osaka International Cancer Institute,

19 Chuo-ku, Osaka, 541-8567, Japan. 
20 8. Research Platform Office, The Institute of Medical Science, The University of Tokyo,

21 Shirokane-dai, Minato-ku, Tokyo 108-8639, Japan.

$23 *$ Corresponding author

24 Jun Nakayama (junakaya@ncc.go.jp, jnakayama.re@gmail.com) and Kentaro Semba

25 (ksemba@waseda.jp), Department of Life Science and Medical Bioscience, School of

26 Advanced Science and Engineering, Waseda University, TWIns, 2-2 Wakamatsu-cho,

27 Shinjuku-ku, Tokyo, 162-8480, Japan, Tel/Fax: +81-3-5369-7320.

\section{Keywords}

30 NIK, Non-canonical NF-אB, Post-translational regulation, in vivo selection, Orthotopic 31 xenograft, Breast cancer

\section{Abstract}

34 Post-translational modifications and mRNA translation are frequently altered in human cancers.

35 However, investigations to understand their roles in the cancer progression mechanism remain

36 insufficient. In this research, we explored protein levels altered by translational or

37 post-translational regulation by analyzing transcriptome and western blotting data of the highly

38 malignant breast cancer cell lines. From these analyses, NIK was found to be upregulated at the

39 protein level to predominantly activate the non-canonical NF-kB pathway in a breast cancer cell

40 line. Furthermore, the increase in NIK protein production was attributed to the dysregulation of 
41 ubiquitin-proteasome system caused by a decrease in the translation of cIAP1. NIK upregulation

42 contributed to tumorigenicity by regulating the expression of inflammatory response-related

43 genes. Collectively, our study suggests that NIK is post-translationally modified and has the

44 potential to be a therapeutic target and diagnostic marker for breast cancer. 
47 Breast cancer is the most common cancer among women worldwide ${ }^{1}$. Although breast 48 cancer mortality has decreased for three decades since the 1990s, the decreasing trend in 49 mortality has slowed in recent years ${ }^{1,2}$. Breast cancer has been traditionally categorized into 50 four molecular subtypes (luminal A, luminal B, human epidermal growth factor receptor type 2 51 (HER2) -enriched, and triple-negative) based on the expression of pathological marker proteins 52 such as HER2, estrogen receptor (ER), and progesterone receptor (PR). In particular, 53 triple-negative breast cancer (TNBC) constitutes approximately 10\% 20\% of breast cancers and 54 is characterized by defects in HER2, ER, and PR expression ${ }^{3,4}$. TNBC presents a relatively poor 55 prognosis, with metastases occurring more frequently than in other subtypes despite the limited 56 molecular therapeutic targets ${ }^{3,5}$. Therefore, it is necessary to explore innovative therapeutic targets by further elucidating the malignant mechanism of TNBC.

In normal cells, signal transductions are tightly regulated through translational and 59 post-translational modification mechanisms, while, in cancer cells, abnormalities in 60 translational and post-translational modifications contribute to their malignancy ${ }^{6,7}$. During the 61 translation process, oncogenic signaling activation, such as through the mTOR and RAS-MAPK 62 pathways, induces an enhancement in eIF4F complex expression and phosphorylation, thereby 63 promoting global translation and contributing to cancer malignancy ${ }^{6}$. On the other hand, 64 unusual tRNA modification and RNA conformations also contribute to cancer progression by 65 promoting the translation of specific genes ${ }^{8-10}$. Post-translational modifications involve a large 66 variety of mechanisms. For example, ubiquitination, one of the major post-translational 67 modifications, has a large number of regulatory functions, including proteostasis, signaling 68 complex assembly, chromatin remodeling, and protein secretion ${ }^{7}$. In particular, protein 
69 degradation by ubiquitination is essential for the regulation of signaling pathways, such as

70 MAPK, NF-кB and PI3K-AKT-mTOR, which play important roles in cell growth and survival ${ }^{7}$.

71 Disruption of ubiquitin-modifying machinery can lead to the malignant transformation of cancer

72 and a variety of other diseases ${ }^{7,11}$. To elucidate mechanisms of cancer malignancy,

73 transcriptome analysis is frequently used, and the development of analysis at single cell level

74 has made it possible to conduct more detailed analysis ${ }^{12}$. However, it is also necessary to

75 conduct analyses integrating transcriptome analysis, translational and post-translational

76 modification evaluations at the protein level.

77 In vivo investigations with cancer cell line-derived models have contributed to the 78 understanding of cancer biology and cancer hallmarks ${ }^{13}$. Animal models enable to analyze the 79 functions of certain genes involved in cancer malignancy and the evaluation of the antitumor 80 properties of preclinical candidate drugs. Among these models, xenograft models have been 81 frequently utilized to assess tumorigenicity at particular organs and metastases to distant organs.

82 Xenograft models are classified into two categories: orthotopic and ectopic. In particular, an

83 orthotopic xenograft $(\mathrm{OX})$ model, which mimics early cancer progression, is appropriate to 84 comprehensively understand tumorigenesis and metastatic mechanisms. Recently we established 85 a cell line, LM05, with high tumorigenic and lung-metastatic properties from a TNBC cell line 86 MDA-MB-231 using an OX model ${ }^{14}$. Interestingly, this cell line showed an different expression 87 profile from that of another lung-metastatic cell line, LM1-2-1, established by tail vein injection 88 (TVI) that corresponds to an ectopic xenograft model ${ }^{14,15}$. However, the molecular mechanisms 89 of high tumorigenic and lung-metastatic properties of LM05 cells have not yet been studied in 90 detail. 
91 In this research, we comparatively analyzed western blot and microarray data of LM05

92 cells to discover their specific activating signals in comparison with parental MDA-MB-231

93 cells and LM1-2-1 cells. As a result, we identified that the non-canonical NF-kB pathway was

94 constitutively activated in LM05 cells via NIK upregulation at the protein level. The aberrant

95 accumulation of NIK due to dysregulation of post-translational modifications promoted

96 tumorigenicity via a cancer-inducing inflammatory response.

Materials and Methods

Cell culture

MDA-MB-231-mSlc7a1-luc2 (parental cells), LM05 and LM1-2-1 cells were established as previously described ${ }^{14}$. These cell lines were cultured in RPMI-1640 (Fujifilm Wako Pure

103 Chemical Corporation, Osaka, Japan) supplemented with 10\% heat-inactivated FBS (Nichirei

104 Biosciences Inc., Tokyo, Japan), 100 U/mL penicillin (Meiji Seika Pharma Co., Ltd., Tokyo, 105 Japan) and $100 \mu \mathrm{g} / \mathrm{mL}$ streptomycin (Meiji-Seika Pharma) at $37^{\circ} \mathrm{C}$ in $5 \% \mathrm{CO}_{2}$. Plat-E packaging 106 cells were kindly provided from T. Kitamura (Institute of Medical Science, University of Tokyo), 107 and TIG-3 cells were kindly provided from B. Shiotani. These cells were cultured in DMEM 108 (Fujifilm Wako Pure Chemical Corporation) supplemented with 10\% heat-inactivated FBS, 100 $109 \mathrm{U} / \mathrm{mL}$ penicillin, and $100 \mu \mathrm{g} / \mathrm{mL}$ streptomycin. 
112 Western blotting was performed as previously described ${ }^{16}$. Cells were lysed in $1 \times$ SDS 113 sample buffer (50 mM Tris-HCl (pH 6.8), 2\% SDS, 5\% 2-mercaptoethanol, $0.1 \%$ BPB, $10 \%$ 114 glycerol) and then boiled at $95^{\circ} \mathrm{C}$ for $5 \mathrm{~min}$. To examine NIK protein level, cells were treated 115 with $10 \mu \mathrm{M}$ MG132 (Peptide Institute, Inc., Osaka, Japan) for 4 hours. To assess cIAP1 protein 116 stability, cells were treated with $10 \mu \mathrm{g} / \mathrm{mL}$ cycloheximide (CHX) (Fujifilm Wako Pure Chemical 117 Corporation). In addition, hypotonic buffer (10 mM HEPES-KOH (pH 7.9), $1.5 \mathrm{mM} \mathrm{MgCl} 2,10$ $118 \mathrm{mM} \mathrm{KCl}$, protease inhibitor cocktail, and $0.15 \mathrm{mM}$ DTT) was used to fractionate nuclear lysates 119 and cytoplasmic lysates. Then, 3× SDS sample buffer was added to each lysate. The lysates 120 were subjected to SDS-PAGE and transferred to PVDF membranes (Millipore, Darmstadt, 121 Germany). Finally, the target proteins were detected using ImmobilonTM Western (Millipore). 122 Information regarding the primary antibodies and secondary antibodies is described in 123 Supplementary Table S1.

Breast cancer cells were lysed in TNE buffer (20 mM Tris- $\mathrm{HCl}(\mathrm{pH} 8.0), 150 \mathrm{mM} \mathrm{NaCl}$, $1 \%$ NP-40, 2 mM EDTA, $25 \mathrm{mM} \mathrm{NaF}, 17.5 \mathrm{mM} \beta$-glycerophosphate, $1 \mathrm{mM} \mathrm{Na} \mathrm{VO}_{4}$, cOmplete (Roche, Basel, Switzerland)). The protein concentrations of the cell lysates were measured by the BCA method (Thermo Fisher Scientific, Waltham, MA, USA). Then, each protein lysate was added to a NIK antibody (\#4994, Cell Signaling Technology, Danvers, MA, USA) or a rabbit IgG antibody (12-370, Millipore) and mixed with rotation overnight. After mixing, $20 \mu \mathrm{L}$ of Protein A Sepharose 4 FF (GE Healthcare Japan, Tokyo, Japan) was added and mixed with rotation at $4{ }^{\circ} \mathrm{C}$ for 3 hours. Subsequently, the supernatant was removed after centrifugation, and the Protein A Sepharose was washed five times with TNE Buffer. Finally, $1 \times$ SDS sample buffer 
135 was added after removing the supernatant followed by heating at $95^{\circ} \mathrm{C}$ for 5 minutes. This

136 extract solution was used as the immunoprecipitation sample.

138 Animal experiments

139 Animal experiments were performed in compliance with the guidelines of the Institute for

140 Laboratory Animal Research, National Cancer Center Research Institute (experimental number:

141 T18-009) and the Animal Committee of Waseda University (accession numbers: WD19-058,

142 2019-A068, WD20-005, 2020-A067, WD21-082, 2021-A074). Female

143 NOD.CB-17-Prkdc-scid/J mice (NOD-SCID, 5-6 weeks old, Charles River Laboratories Japan,

144 Inc., Kanagawa, Japan) were used for the orthotopic xenograft and tail vein injection models.

145 The methods for establishing the xenograft metastasis model and tail vein injection model and

146 performance of bioluminescence imaging were previously described ${ }^{14}$. For orthotopic

147 xenografts, a total of $1.0 \times 10^{6}$ cancer cells in $10 \mu \mathrm{l}$ of D-PBS(-) (Fujifilm Wako Pure Chemical

148 Corporation) were implanted into the fourth fat pad of each mouse using a 28-gauge syringe.

149 When the primary tumor volumes reached $300 \mathrm{~mm}^{3}$, they were resected under anesthesia with

$150 \quad 2.5 \%$ isoflurane (Fujifilm Wako Pure Chemical Corporation). After the primary tumors were

151 resected, mice were periodically monitored for metastasis formation using an in vivo imaging

152 system (IVIS) -Lumina XRMS (Perkin-Elmer, Waltham, MA, USA) for 2 weeks. For the tail

153 vein injection model, a total of $5.0 \times 10^{5}$ cancer cells in $100 \mu 1$ of D-PBS(-) (Wako Pure

154 Chemical Industries) were injected into the tail vein of each mouse using a 27-gauge needle. To

155 monitor these xenograft mice, $200 \mu \mathrm{L}$ of D-luciferin (15 mg/mL) (Gold Biotechnology, Inc., St.

156 Louis, MO, USA) was injected intraperitoneally into each mouse, and bioluminescence imaging

157 was performed with the IVIS every week. In addition, the bioluminescence of the lung 
metastatic tissue was measured ex vivo using the IVIS after intraperitoneal administration of $200 \mu \mathrm{L}$ of D-luciferin to assess the metastatic potential of the cancer cells.

\section{Histochemical analyses}

The dissected primary tumors and lung metastasis tissues from the orthotopic mouse model were fixed with $4 \%$ paraformaldehyde-PBS and embedded in paraffin. The paraffin-embedded human breast cancer cells with a breast tissue microarray (BC081116d, US Biomax, MD, USA) were baked for 2 hours at $60^{\circ} \mathrm{C}$ before proceeding with the following steps. The paraffin sections were deparaffinized and rehydrated in xylene, a graded ethanol series that decreased stepwise from $100 \%$ to $50 \%$, and distilled water.

For immunohistochemistry (IHC) analysis, antigen retrieval was performed in $10 \mathrm{mM}$ citrate buffer ( $\mathrm{pH} \mathrm{6.0)}$ at $120^{\circ} \mathrm{C}$ or $95^{\circ} \mathrm{C}$ for 20 minutes. After cooling to room temperature, the endogenous peroxidase activity was blocked with $3 \% \mathrm{H}_{2} \mathrm{O}_{2}$ in $10 \%$ methanol for $30 \mathrm{~min}$. Then, these sections were incubated in $2.5 \%$ normal horse serum (Vector Laboratories, CA, USA) for 1hour at room temperature. The specimens were incubated with primary antibodies against a-SMA (A2547, Sigma-Aldrich Co., MO, USA), Ki-67 (ab16667, Abcam, Cambridge, UK), CD206 (AF2535, R\&D Systems, Inc., MN, USA), CAM5.2 (\#349205, BD Bioscience, CA, USA), and NIK (HPA027269, Sigma-Aldrich Co.) at $4^{\circ} \mathrm{C}$ overnight. Then, the sections were incubated with the secondary antibody solutions for 1 hour at room temperature. The sections were stained with an ImmPACT® $\mathrm{DAB} \mathrm{EqV}$ substrate kit (Vector Laboratories) followed by staining with hematoxylin, dehydration and mounting. Images were acquired with a BZ-X700 microscope (Keyence Corporation, Osaka, Japan) and analyzed using the image analysis 
application for BZ-X700 (Keyence Corporation) and ImageJ software (National Institutes of 181 Health).

For hematoxylin and eosin (HE) staining, deparaffinized and rehydrated sections were stained in Mayer's hematoxylin solution (Muto Pure Chemicals Co., Tokyo, Japan) for 10min. Then, the sections were soaked in $0.1 \%$ saturated lithium carbonate at $37^{\circ} \mathrm{C}$ for $5 \mathrm{~min}$. After washing in distilled $\mathrm{H} 2 \mathrm{O}$, the sections were stained with eosin solution (Muto Pure Chemicals

186 Co.) for $10 \mathrm{~min}$. Then, the sections were immediately washed with $100 \%$ ethanol, $90 \%$ ethanol 187 and xylene. Finally, the sections were mounted, and images were acquired with a BZ-X700 microscope.

For terminal deoxynucleotidyl transferase dUTP nick end labeling (TUNEL) staining,

190 deparaffinized and rehydrated sections were stained with a TUNEL Assay Kit (ab206386, Abcam) according to the manufacturer's protocols. After staining with hematoxylin, dehydration and mounting, images were acquired with a BZ-X700 microscope (Keyence 193 Corporation).

Total RNA was extracted with QIAzol (Qiagen, CA, USA) according to the manufacturer's 197 protocol. After purification of the total RNA, the quantity and quality of the RNA were evaluated with a Nanodrop ND-1000 spectrophotometer (Thermo Fisher Scientific). cDNA libraries for RNA sequencing were established from total RNA using NEBNext Poly(A) mRNA 200 Magnetic Isolation Module (New England Biolabs, MA, USA) to select poly-A mRNA 
202 Set V2.0 (MGITech Co. Shenzhen, China). Paired-end sequencing with a lead length of 150

203 bases was performed on a DNBSEQ-G400 (MGI tech) platform following the manufacturer's 204 instructions.

205 The raw sequence data (fastq files) were trimmed to remove adapter sequences by 206 Trim-Galore (v0.6.4), and then, the trimmed read data were mapped on GRCh38/hg38 207 (GENCODE) using HISAT2 (v2.2.0). After the SAM files were converted Bam files using 208 SAMtool (v1.10), the gene expression FPKM was quantified by StringTie (v2.1.2). Then, read 209 count matrices were generated for each gene by prepDE.py. After removing low-expression 210 genes with less than 10 counts per million (CPM), differentially expressed genes were identified 211 using edgeR in R. Statistical cutoffs based on a p-value $<0.05$ and a log fold change (Log2(FC)) $212 \pm 1$ were used to filter DEGs between LM05-shGFP cells and LM05-shNIK cells. The $\mathrm{z}$ scores 213 of these DEGs were calculated by gene filtering, and hierarchical clustering heatmaps were 214 created using pheatmap in R (v3.6.2). For pathway analysis, the expression data were analyzed 215 with gene set enrichment analysis (GSEA; https://www.gsea-msigdb.org/gsea/index.jsp) and

216 ingenuity pathway analysis

217 (IPA; https://digitalinsights.qiagen.com/products-overview/discovery-insights-portfolio/analysis

218 -and-visualization/qiagen-ipa/).

All statistical analyses were performed with GraphPad Prism 7 (GraphPad Software, San Diego, CA, USA). Statistical results are presented as the mean \pm SEM. Welch's $t$ test and one-way or two-way ANOVA were employed for comparisons between two or three groups of 224 data. 
RNA-seq data were submitted to the NCBI GEO database under accession number

GSE182261. The microarray expression data of the breast cancer cells that can generate lung metastases were obtained from our previous research ${ }^{14}$. These expression data and other supporting data of this research are available from the corresponding authors upon reasonable request.

Other experimental methods are included in Supplementary Methods.

\section{Results}

238 We previously established two lung metastatic breast cancer cell lines (LM05 and 239 LM1-2-1) ${ }^{14}$. LM05 cells were established from MDA-MB-231 cells (parental cells) by two 240 cycles of the generation of OX and subsequent extraction from the lung metastatic tissue (Fig. 241 1A). On the other hand, LM1-2-1 cells were established from parental cells by two cycles of 242 TVI and subsequent extraction from the lung metastatic tissue (Fig. 1A). LM05 cells have a 243 high potential for tumorigenicity in the fat pads compared with the parental cells and LM1-2-1 244 cells (Fig. 1B) ${ }^{14}$. In previous studies, transcriptome analysis of these highly malignant cell lines 245 was performed as a conventional research strategy ${ }^{17-19}$. In this research, considering the 246 importance of translational and post-translational modifications in cancer malignancy, we 
investigated proteins that showed changes in amount and/or modification after the translation

248 process by comparison between western blotting and the microarray expression data to discover

249 the factors that enhanced tumorigenicity. We employed LM1-2-1 cells as the comparison subject

250 to search for specific factors in LM05 cells. The analysis results showed that the amounts of 251 mRNA and protein related with EGFR-MAPK, AKT-mTOR and JAK-STAT pathways were not 252 changed (Fig. 1C, D), although the protein level of NF-kB2 (p52) increased (Fig. 1D). The 253 activation of NF- $\mathrm{KB}$ signaling results in nuclear translocation of the NF- $\mathrm{kB}$ family of proteins:

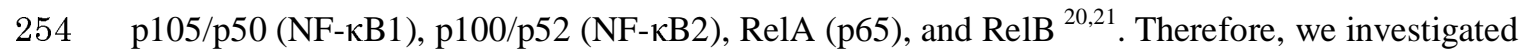
255 the nuclear translocation of the NF- $\mathrm{kB}$ family proteins by western blotting to evaluate the

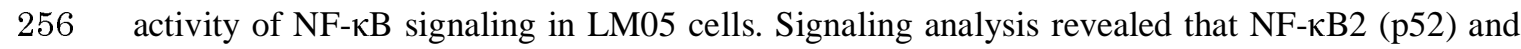
257 RelB increased in the nuclear fraction of LM05 cells compared with the parental cells and 258 LM1-2-1 cells (Fig. 1E). On the other hand, p50 and RelA in the nuclear fraction were 259 unchanged in LM05 cells (Fig. 1E). Since p52 and RelB are classified as part of the 260 non-canonical NF-kB pathway, we examined the amounts of NF-kappa- $\beta$-inducing kinase 261 (NIK), a key regulator of the non-canonical NF-kB pathway. NIK is rapidly degraded by ubiquitin-proteasome system after ubiquitinated with TRAF2-TRAF3-cIAP1/2 complex ${ }^{22,23}$.

263 Therefore, we investigated the amounts of NIK protein in the presence of a proteasome inhibitor, 264 MG132. As a result, NIK was found to be increased at the protein level even though NIK mRNA 265 expression was not significantly changed in LM05 cells (Fig. 1F). These results indicated that 266 the non-canonical NF-kB pathway was constitutively activated in LM05 cells via NIK 267 upregulation at the protein level. 
270 NIK is constantly degraded in the normal state by the TRAF2-TRAF3-cIAP1/2 complex in 271 the ubiquitin-proteasome system ${ }^{22,23}$. We therefore examined the ubiquitination of NIK by 272 immunoprecipitation with a NIK antibody. Ubiquitination of NIK was found to be decreased in 273 LM05 cells compared with parental cells (Fig. 2A). Next, we examined the amounts of cIAP1, 274 which acts as an E3 ligase to ubiquitinate NIK ${ }^{22,23}$. cIAP1 was found to be decreased at the 275 protein level even though cIAP1 mRNA expression was unchanged compared to the parental 276 and LM05 cells (Fig. 2B). Notably, NIK upregulation in LM05 cells was suppressed by cIAP1 277 overexpression (Supplementary Fig. S1A). From these results, the mechanism of NIK 278 upregulation in LM05 cells was dependent on cIAP1 reduction. Next, we evaluated the protein 279 stability of cIAP1 by cycloheximide chase analysis. These results demonstrated that the cIAP1 280 reduction at the protein level was not ascribed to protein degradation in LM05 cells (Fig. 2C). In 281 previous studies, it was established that nascent cIAP1 production was occasionally regulated 282 by translational mechanisms ${ }^{24-26}$. Hence, we evaluated the production of the nascent cIAP1 283 protein pulse-labeled with L-homopropargylglycine (HPG) as a methionine analog conjugated 284 to a biotin tag using a click reaction. The results showed that HPG-labeled cIAP1 protein 285 production decreased in LM05 cells compared with parental cells (Fig. 2D). All of these results 286 indicated that the downregulation of cIAP1 translation caused NIK stabilization, which 287 contributed to the constitutive activation of the non-canonical NF- $\mathrm{BB}$ pathway.

NIK knockdown reduced colony formation activity in LM05 cells

To determine the contribution of NIK to cancer malignancy, we characterized the 291 phenotype of LM05 cells after NIK knockdown in vitro (Fig. 3A). NIK knockdown cells 292 depressed the non-canonical NF-kB pathway by suppressing the nuclear localization of p52 and 
293 RelB (Fig. 3B). We next evaluated cell proliferation and anchorage-independent growth with a

294 soft agar assay. NIK expression did not affect cell growth in planar culture (Fig. 3C), although

295 the colony formation activity was decreased by NIK knockdown (Fig. 3D). We therefore

296 assessed the contribution of NIK knockdown to cancer stemness, since NIK is known to be a

297 supportive factor of cancer stemness from the results of previous studies ${ }^{27,28}$. Notably, NIK

298 knockdown did not bring about changes in the stemness population rate (Fig. 3E) or sphere

299 formation in the mammosphere culture assay (Fig. 3F). From these results, NIK upregulation in

300 LM05 cells contributed to colony formation but not cancer stemness.

301

Upregulation of NIK contributed to tumorigenicity in LM05 cells

To investigate whether NIK upregulation contributes to the inherent high tumorigenicity and potential of LM05 cells to metastasize to the lungs, NIK knockdown cells were orthotopically injected into the mammary fat pats of NOD-SCID mice. NIK knockdown in

306 LM05 cells significantly decreased the primary tumor weights and volumes (Fig. 4A and B). In addition, the reduction in tumorigenicity caused by NIK knockdown was partially rescued by recovering the ectopic expression of NIK (Supplementary Fig. S2A, B and C). In addition, we examined the effects of NIK knockdown on metastatic potential by performing a metastasis assay in an orthotopic xenograft model. To remove the effects from tumorigenicity reduction created by NIK knockdown, we uniformly resected the primary tumors after they reached a size of $300 \mathrm{~mm}^{3}$. Under these conditions, NIK knockdown did not affect the potential of LM05 cells to form lung metastases (Fig. 4C). Furthermore, IHC analysis of lung metastases using an anti-CAM5.2 antibody, a human-specific cytokeratin, indicated that there were no differences in 
results indicated that NIK upregulation facilitated the inherent tumorigenicity but not lung metastatic potential of LM05 cells.

Next, we performed immunohistochemical staining of the primary tumor to investigate the role of NIK in the primary tumor. The number of TUNEL-positive cells (apoptosis marker) increased in the primary tumor after NIK knockdown (Fig. 4E). In addition, the $\alpha$-SMA-positive region (a cancer-associated fibroblast (CAF) marker) decreased in the primary tumors after NIK knockdown (Fig. 4E). Therefore, we utilized a co-culture system in a Boyden chamber to investigate the effects of NIK on the human fibroblast cell line TIG-3. From the experimental results, it was found that NIK knockdown suppressed the attraction of TIG-3 cells to the cancer cells in the co-culture system (Fig. 4F). On the other hand, $\alpha$-SMA protein in TIG-3 cells did not change when co-cultured with control and NIK knockdown cells (Fig. 4G), indicating that NIK apoptosis of the tumor cells and decreased induction of CAFs.

To determine the NIK-related pathway and genes that contribute to the phenotypes altered by NIK knockdown (Fig. 4A, B and E), we performed transcriptome analysis by RNA-seq. We extracted the differentially expressed genes (DEGs) between LM05-shGFP cells and LM05-shNIK cells using the following criteria: FDR $<0.05$ (Benjamini-Hochberg method) and $\log$ fold change $\left(\log _{2}(\mathrm{FC})\right) \pm 1$ (Fig. 5A). To determine the NIK-related pathways, the common DEGs in the NIK knockdown cells were analyzed by gene set enrichment analysis (GSEA) and ingenuity pathway analysis (IPA). GSEA using the MSigDB Hallmark gene set collection 
339 revealed that the genes with decreased expression in the NIK knockdown cells were

340 significantly enriched for pathways related to inflammation, interferon response, and TNF $\alpha$

341 signaling via NF-кB (Fig. 5B, Supplementary Table S2). Similarly, pathways related to the 342 interferon response and TNF secretion were suppressed in NIK knockdown cells using Gene

343 Ontology gene sets (Supplementary Table S3). Furthermore, upstream regulator analysis by IPA 344 contained components of NF- $\mathrm{KB}$ signaling as an upstream regulator of the downregulated 345 pathway (Supplementary Fig. S3A). These transcriptome analyses showed that the 346 inflammatory-related genes regulated by the NF-kB pathway were suppressed in LM05 NIK 347 knockdown cells. Indeed, NIK knockdown decreased the expression of IL6 and CXCL1, which 348 are important for the migration of fibroblasts (Fig. 5C) ${ }^{29,30}$. Furthermore, BIRC3 expression, 349 which contributes to apoptosis resistance, was markedly reduced by NIK knockdown (Fig. 5C)

$350{ }^{31,32}$. Based on these results, the reduction in BIRC3 expression and fibroblast-attracted 351 cytokines and chemokines caused by NIK knockdown inherently result in decreased 352 tumorigenicity.

355 We then investigated the relationship between NIK production and breast cancer malignancy. 356 We characterized NIK production by immunohistochemistry in a tissue microarray from breast 357 cancer patients (Fig 6A). IHC analysis showed that NIK production was significantly increased 358 in stage I-III patients compared with normal breast tissues (Supplementary Table S4, Fig. 6B). 359 In addition, NIK protein production was not correlated with the expression scores of breast 360 cancer marker genes such as ER, PR, and HER2 (Supplementary Fig. S4A, B and C). From 
these results, NIK has the potential to be used as a diagnostic marker across other breast cancer subtypes.

\section{Discussion}

Constitutive activation of the non-canonical NF- $\mathrm{kB}$ pathway has been reported in breast lines have shown increased NIK mRNA expression by epigenetic dysregulation of NIK gene, which induces constitutive activation of the non-canonical pathway ${ }^{35,36}$. On the other hand, certain multiple myeloma cell lines have genetic defects in TRAF2/3 and cIAP1/2 ${ }^{33,34}$, which are repressors of the non-canonical pathway. However, constitutive activation of the

372 non-canonical pathway due to reduced translation of cIAP1, as revealed in this study, is a novel 373 activation mechanism of the non-canonical pathway in breast cancer cell lines (Fig. 6C). In 374 previously, the activation of the non-canonical NF- $\mathrm{kB}$ pathway by NIK upregulation contributes 375 to the enhancement in cell proliferation and maintenance of cancer stemness properties via the 376 NOTCH pathway, especially in basal-like breast cancer cell lines ${ }^{27,35}$. Although claudin-low cell lines exhibit high levels of NF- $\mathrm{B}$ activation with increased NIK mRNA expression ${ }^{35,36}$, the function of NIK in tumorigenesis and metastasis has not been investigated. Our results suggested that NIK upregulation contributed to tumorigenicity by attracting CAFs and inhibiting apoptosis through regulation of the expression of genes related to inflammatory responses in LM05 cells, which is classified as the claudin-low subtype (Fig. 6C). Therefore,

382 there are novel function and aberrant accumulation mechanism of NIK in breast cancer 383 malignancy that differs from the conventional researches. 
From our results, a reason for the reduction of tumorigenicity by NIK knockdown is the expansion of the apoptotic area in the primary tumor. In addition, our results suggest that the decrease in BIRC3 expression, which has antiapoptotic properties, and CAFs may contribute to the increase in the apoptotic area. CAFs have been reported to contribute to the enhancement of tumor malignancy by promoting the antiapoptotic potential and chemoresistance of cancer cells 37,38. It is possible that a reduction in the number of CAFs in tumors by NIK knockdown contributes to the decline in the antiapoptotic potency of cancer cells. CXCL12/SDF-1 secreted by CAFs contributes to tumor growth and anti-apoptosis in cancer cells via CXCR $4{ }^{39,40}$. Indeed, the expression of CXCR4 was reduced in NIK knockdown cells; thus, there was a deficiency in the positive feedback through this paracrine mechanism. Therefore, NIK plays an important role in the interaction with stromal cells in the cancer microenvironment.

NIK is crucial for the maintenance of various tissue functions, including the immune system, bone formation, the kidneys, the liver, glucose homeostasis and hematopoiesis ${ }^{41}$. Therefore, abnormal NIK activation has been implicated in a variety of autoimmune diseases, such as systemic lupus erythematosus, acute kidney injury and cancer ${ }^{41}$. Recently, exploratory studies of NIK inhibitors have expanded ${ }^{41}$. NIK inhibitors have been developed by modifying inhibitors of similar kinase family members and conventional NIK inhibitors based on structure-activity relationships and docking simulations using NIK crystallographic data ${ }^{42,43}$.

402 These studies have identified that NIK inhibitors have therapeutic effects in certain mouse 403 models of inflammatory hepatic diseases and systemic lupus erythematosus ${ }^{44,45}$. In addition, 404 mangiferin, which is a natural compound with NIK inhibitory activity, has been reported to 405 inhibit tumorigenesis and metastatic potential in melanoma cell lines ${ }^{46}$. However, the efficacy 406 of these NIK inhibitors against other types of cancer is unclear; thus, verification of the activity 407 of these NIK inhibitors is needed to examine this possibility. In contrast, inactivating NIK 
mutations have been shown to cause immunodeficiency, such as decreased numbers of mature B

409 cells and $\mathrm{T}$ cells their functional impairment ${ }^{47}$. NIK plays an important role in antitumor

410 immunity by regulating metabolism in cytotoxic $\mathrm{CD}^{+} \mathrm{T}$ cells ${ }^{48}$. Therefore, it is necessary to

411 develop NIK inhibitors through further comprehension of the reciprocal NIK functions to avoid

412 interfering with the homeostatic role that NIK plays in the body. Our experimental results

413 indicated that NIK knockdown critically affected tumorigenicity rather than the potential to

414 form lung metastases in LM05 cells. From this result, we hypothesized that NIK may play an

415 important role in tumorigenesis mechanisms in the mammary gland. To address this hypothesis,

416 we evaluated the proliferative potential of parental and LM05 cells in lung tissue using tail vein

417 injection. The results showed that there was no significant difference in the proliferative

418 potential of the lung tissues between the parental and LM05 cells (Supplementary Fig. S5A). In

419 addition, LM05 cells were repeatedly xenografted into the mammary gland and grown at this

420 site. Thus, the enhanced tumorigenicity of LM05 is probably mammary gland-specific. Indeed,

421 TWEAK and RANKL, ligands of the non-canonical NF- $\mathrm{B}$ pathway, are expressed and have 422 important functions in mammary gland development and tumorigenesis ${ }^{49-52}$. In short, LM05

423 cells may be more susceptible to the activation of the non-canonical pathway by these ligands in 424 breast tissue because of the accumulation of NIK proteins. Meanwhile, we were not able to 425 identify the genes that contribute to the enhanced potential of LM05 cells to metastasize to the 426 lungs. However, we identified a group of genes that were upregulated in LM05 cells compared 427 to the parental strain but not downregulated after NIK knockdown, including known lung 428 metastasis regulatory genes such as IL13RA2, TNS1, and EMP1 18,53,54 (Supplementary Fig. 429 S6A). Further studies of those genes may reveal the NIK-independent metastasis enhancement 430 mechanism in LM05 cells. 
431 In conclusion, our results demonstrated a novel role and mechanism of NIK after its 432 upregulation during breast cancer progression. In addition, the amount of NIK protein was 433 increased in tumor tissues compared to normal tissues; thus, NIK has the potential to be a 434 diagnostic marker of breast cancer. Elucidating the functions and regulatory mechanisms of NIK 435 can lead to a deeper understanding of NIK as a potential biomarker or therapeutic target in 436 breast cancer. 
438 We thank Dr. Bunsyo Shiotani (National Cancer Center Research Institute) for providing the

439 TIG-3 cells. We are also grateful to our laboratory member for worthwhile discussion and 440 technical advice. Y.H. was supported by doctoral scholarships of the Futaba foundation.

441 Schematics in figures were made using an academic license of BioRender.com.

Funding

444 This research was supported by Japan Society for the Promotion of Science ((Grant Numbers

445 18K16269: Grant-in-Aid for Early-Career Scientists to J.N., Grant Number 20J01794 to J.N.

446 and 21K15562 to J.N.: Grant-in-Aid for JSPS Fellows), Foundation for Promotion of Cancer

447 Research in Japan, the Fukushima Translational Research Project and the Japan Biological 448 Informatics Consortium (JBiC) to S.W. and K.S.

\section{Authors' contributions}

J.N. and K.S. conceived and designed the study. Y.H. and J.N. performed the analyses and the experiments. M.Y., M.M., S.W., S.H., J.I. and Y.Y. interpreted the data. Y.H. wrote the original paper. J.N., Y.Y. and K.S. revised the manuscripts. All authors reviewed and edited the manuscript.

\section{Conflicts of interest}

The authors declare that they have no competing interests. 


\section{$458 \quad$ References}

459 1. Siegel, R. L., Miller, K. D. \& Jemal, A. Cancer statistics, 2020. CA. Cancer J. Clin. 70, $460 \quad 7-30(2020)$.

461 2. Liu, H. et al. Global, regional, and national mortality trends of female breast cancer by risk 462 factor, 1990-2017. BMC Cancer 21, 1-10 (2021).

3. Foulkes, W. D., Smith, I. E. \& Reis-Filho, J. S. Triple-Negative Breast Cancer. N. Engl. J. Med. 363, 1938-1948 (2010).

4. Acheampong, T., Kehm, R. D., Terry, M. B., Argov, E. L. \& Tehranifar, P. Incidence Trends of Breast Cancer Molecular Subtypes by Age and Race/Ethnicity in the US From

5. Dent, R. et al. Triple-negative breast cancer: Clinical features and patterns of recurrence. Clin. Cancer Res. 13, 4429-4434 (2007).

6. Micalizzi, D. S., Ebright, R. Y., Haber, D. A. \& Maheswaran, S. Translational Regulation of Cancer Metastasis. Cancer Res. 81, 517-524 (2021). therapy. Nat. Rev. Cancer 18, 69-88 (2018).

8. Phung, B. et al. The X-Linked DDX3X RNA Helicase Dictates Translation Reprogramming and Metastasis in Melanoma. Cell Rep. 27, 3573-3586.e7 (2019). cancer progression. Cell 165, 1416-1427 (2016). 
478 10. Rapino, F. et al. Codon-specific translation reprogramming promotes resistance to targeted $479 \quad$ therapy. Nature 558, 605-609 (2018).

11. Popovic, D., Vucic, D. \& Dikic, I. Ubiquitination in disease pathogenesis and treatment. Nat. Med. 20, 1242-1253 (2014).

12. Fan, J., Slowikowski, K. \& Zhang, F. Single-cell transcriptomics in cancer: computational challenges and opportunities. Exp. Mol. Med. 52, 1452-1465 (2020).

13. Gengenbacher, N., Singhal, M. \& Augustin, H. G. Preclinical mouse solid tumour models: Status quo, challenges and perspectives. Nat. Rev. Cancer 17, 751-765 (2017).

14. Nakayama, J., Ito, E., Fujimoto, J., Watanabe, S. \& Semba, K. Comparative analysis of gene regulatory networks of highly metastatic breast cancer cells established by orthotopic

15. Nakayama, J. et al. The In Vivo Selection Method in Breast Cancer Metastasis. Int. J. Mol. transplantation and intra-circulation injection. Int. J. Oncol. 50, 497-504 (2017).

16. Kuroiwa, Y. et al. Proliferative Classification of Intracranially Injected HER2-positive Breast Cancer Cell Lines. Cancers (Basel). 12, 1811 (2020).

17. Kang, Y. et al. A multigenic program mediating breast cancer metastasis to bone. Cancer Cell 3, 537-549 (2003).

18. Minn, A. J. et al. Genes that mediate breast cancer metastasis to lung. Nature 436, 518-524 (2005).

19. Bos, P. D. et al. Genes that mediate breast cancer metastasis to the brain. Nature 459, 1005-1009 (2009). 
20. Hoesel, B. \& Schmid, J. A. The complexity of NF-kB signaling in inflammation and cancer. Mol. Cancer 12, 86 (2013).

21. Huxford, T. \& Ghosh, G. A structural guide to proteins of the NF-kappaB signaling module. Cold Spring Harb. Perspect. Biol. 1, 1-17 (2009).

22. Zarnegar, B. J. et al. Noncanonical NF- $\mathrm{kB}$ activation requires coordinated assembly of a regulatory complex of the adaptors cIAP1, cIAP2, TRAF2 and TRAF3 and the kinase NIK. Nat. Immunol. 9, 1371-1378 (2008).

23. Morrison, M. D., Reiley, W., Zhang, M. \& Sun, S.-C. An Atypical Tumor Necrosis Factor (TNF) Receptor-associated Factor-binding Motif of B Cell-activating Factor Belonging to the TNF Family (BAFF) Receptor Mediates Induction of the Noncanonical NF- $\kappa B$ Signaling Pathway. J. Biol. Chem. 280, 10018-10024 (2005).

24. Van Eden, M. E., Byrd, M. P., Sherrill, K. W. \& Lloyd, R. E. Translation of cellular inhibitor of apoptosis protein 1 (c-IAP1) mRNA is IRES mediated and regulated during cell stress. RNA 10, 469-81 (2004).

25. Graber, T. E., Baird, S. D., Kao, P. N., Mathews, M. B. \& Holcik, M. NF45 functions as an IRES trans-acting factor that is required for translation of cIAP1 during the unfolded protein response. Cell Death Differ. 17, 719-729 (2010).

26. Faye, M. D. et al. IGF2BP1 controls cell death and drug resistance in rhabdomyosarcomas by regulating translation of cIAP1. Oncogene 34, 1532-1541 (2015).

27. Yamamoto, M. et al. NF-kB non-cell-autonomously regulates cancer stem cell populations in the basal-like breast cancer subtype. Nat. Commun. 4, (2013). 
520 28. Vazquez-Santillan, K. et al. NF-kappaB-inducing kinase regulates stem cell phenotype in $521 \quad$ breast cancer. Sci. Rep. 6, 37340 (2016).

522
29. Hendrayani, S. F., Al-Khalaf, H. H. \& Aboussekhra, A. The cytokine il-6 reactivates breast stromal fibroblasts through transcription factor STAT3-dependent up-regulation of the RNA-binding protein AUF1. J. Biol. Chem. 289, 30962-30976 (2014).

30. Buskermolen, J. K., Roffel, S. \& Gibbs, S. Stimulation of oral fibroblast chemokine receptors identifies CCR3 and CCR4 as potential wound healing targets. J. Cell. Physiol. 232, 2996-3005 (2017).

31. Petersen, S. L., Peyton, M., Minna, J. D. \& Wang, X. Overcoming cancer cell resistance to Smac mimetic induced apoptosis by modulating cIAP-2 expression. Proc. Natl. Acad. Sci. U. S. A. 107, 11936-11941 (2010).

32. Bertrand, M. J. M. et al. cIAP1 and cIAP2 Facilitate Cancer Cell Survival by Functioning as E3 Ligases that Promote RIP1 Ubiquitination. Mol. Cell 30, 689-700 (2008).

33. Annunziata, C. M. et al. Frequent Engagement of the Classical and Alternative NF- $\kappa \mathrm{B}$ Pathways by Diverse Genetic Abnormalities in Multiple Myeloma. Cancer Cell 12, $115-130$ (2007).

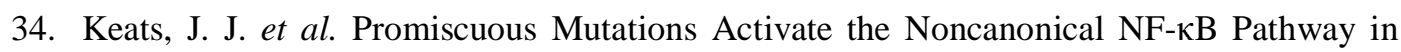
Multiple Myeloma. Cancer Cell 12, 131-144 (2007).

35. Yamaguchi, N. et al. Constitutive activation of nuclear factor- $\kappa \mathrm{B}$ is preferentially involved in the proliferation of basal-like subtype breast cancer cell lines. Cancer Sci. 100, 1668-1674 (2009). 
541 36. Yamamoto, M. et al. Epigenetic alteration of the NF-kB-inducing kinase (NIK) gene is

542 involved in enhanced NIK expression in basal-like breast cancer. Cancer Sci. 101, $543 \quad 2391-2397(2010)$.

544 37. Su, S. et al. CD10+GPR77+ Cancer-Associated Fibroblasts Promote Cancer Formation and $545 \quad$ Chemoresistance by Sustaining Cancer Stemness. Cell 172, 841-856.e16 (2018).

546 38. Weigel, K. J. et al. CAF-secreted IGFBPs regulate breast cancer cell anoikis. Mol. Cancer $547 \quad$ Res. 12, 855-866 (2014).

548 39. Orimo, A. et al. Stromal fibroblasts present in invasive human breast carcinomas promote 549 tumor growth and angiogenesis through elevated SDF-1/CXCL12 secretion. Cell 121, $550 \quad 335-348(2005)$.

40. Wang, C. et al. Sulfatase 2-Induced Cancer-Associated Fibroblasts Promote Hepatocellular Carcinoma Progression via Inhibition of Apoptosis and Induction of

41. Valiño-Rivas, L. et al. NIK as a Druggable Mediator of Tissue Injury. Trends Mol. Med. Epithelial-to-Mesenchymal Transition. Front. Cell Dev. Biol. 9, 1-20 (2021).

42. Blaquiere, N. et al. Scaffold-Hopping Approach to Discover Potent, Selective, and Efficacious Inhibitors of NF-Î B Inducing Kinase. J. Med. Chem. 61, 6801-6813 (2018).

43. Pippione, A. C. et al. N-Acetyl-3-aminopyrazoles block the non-canonical NF-kB cascade by selectively inhibiting NIK. Medchemcomm 9, 963-968 (2018). 
44. Ren, X. et al. A small-molecule inhibitor of NF- $\kappa B$-inducing kinase (NIK) protects liver from toxin-induced inflammation, oxidative stress, and injury. FASEB J. 31, 711-718 (2017).

45. Brightbill, H. D. et al. NF- $\mathrm{BB}$ inducing kinase is a therapeutic target for systemic lupus erythematosus. Nat. Commun. 9, 1-14 (2018).

46. Takeda, T. et al. Mangiferin, a novel nuclear factor kappa B-inducing kinase inhibitor, suppresses metastasis and tumor growth in a mouse metastatic melanoma model. Toxicol. Appl. Pharmacol. 306, 105-112 (2016).

47. Willmann, K. L. et al. Biallelic loss-of-function mutation in NIK causes a primary immunodeficiency with multifaceted aberrant lymphoid immunity. Nat. Commun. 5, (2014).

48. Gu, M. et al. NF-kB-inducing kinase maintains $\mathrm{T}$ cell metabolic fitness in antitumor immunity. Nat. Immunol. 22, 193-204 (2021).

49. Fata, J. E. et al. The Osteoclast Differentiation Factor Osteoprotegerin-Ligand Is Essential for Mammary Gland Development. Cell 103, 41-50 (2000).

50. Schramek, D. et al. Osteoclast differentiation factor RANKL controls development of progestin-driven mammary cancer. Nature 468, 98-102 (2010).

51. Michaelson, J. S. et al. Tweak induces mammary epithelial branching morphogenesis. Oncogene 24, 2613-2624 (2005). 
579 52. Yin, X. et al. RG7212 anti-TWEAK mAb inhibits tumor growth through inhibition of

580 tumor cell proliferation and survival signaling and by enhancing the host antitumor

581 immune response. Clin. Cancer Res. 19, 5686-5698 (2013).

582 53. Chang, K.-C. et al. MaTAR25 lncRNA regulates the Tensin1 gene to impact breast cancer 583 progression. Nat. Commun. 11, 6438 (2020).

584 54. Ahmat Amin, M. K. B. et al. Epithelial membrane protein 1 promotes tumor metastasis by 585 enhancing cell migration via copine-III and Rac1. Oncogene 37, 5416-5434 (2018). 


\section{$587 \quad$ Figure legends}

588 Figure 1. The non-canonical NF-אB pathway was constitutively activated in LM05 cells via 589 upregulation of NIK at the protein level.

590 A. Schematic representation of the in vivo selection process using an orthotopic xenograft (OX) 591 and tail vein injection (TVI). Luciferase-expressing MDA-MB-231 (parental cells) cells were transplanted into NOD-SCID mice by OX or TVI. Subsequently, lung metastatic cells were collected and established from lung tissue with metastases. These cells were reinjected into

594 NOD-SCID mice using the same xenograft model to concentrate these cells with higher lung

595 metastatic activity. B. Representative in vivo bioluminescent images (upper) and tumor growth 596 curves (lower, $\mathrm{n}=4$ per group, two-way ANOVA followed by Tukey's multiple comparison test) 597 of NOD-SCID mice orthotopically injected with parental or LM05 cells at 0 week and 5 weeks. 598 C. Integrative signaling analysis of LM05 cells analyzed by microarray expression data. The 599 microarray expression data results are shown as a heatmap of the $\log _{2}$-fold change in each cell 600 compared with the parental cells. D. Integrative signaling analysis of LM05 cells analyzed by 601 western blotting. The western blotting results had four evaluation criteria (yellow: upregulated, 602 green: downregulated, and gray: unchanged protein production levels compared with parental

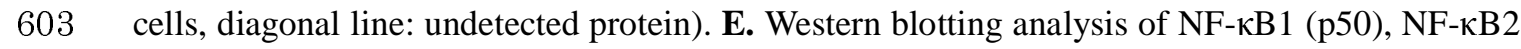
604 (p100/52), RelA and RelB in whole cells and the nuclear and cytoplasmic extracts of the 605 parental, LM05, and LM1-2-1 cells. F. Western blotting (upper) and qRT-PCR (lower, n=3, 606 Welch's t test) results of NIK protein and mRNA in the parental, LM05, and LM1-2-1 cells. For 607 western blotting, all cell lines were either untreated or treated with MG132 (10 $\mu \mathrm{M}$ for $4 \mathrm{hr}$ ). All 608 data are representative of three independent experiments and are shown as the mean \pm SEM. NS, 609 not significant. $* \mathrm{P}<0.05$. 
611 Figure 2. Downregulation of cIAP1 translation caused NIK stabilization, which 612 contributed to constitutive activation of the non-classical NF-kB pathway.

613 A. Western blotting analysis of ubiquitinated NIK in HA-tagged ubiquitin (HA-UB)-expressing

614 parental or LM05 cells after treatment with $10 \mu \mathrm{M}$ MG132 for $4 \mathrm{hr}$. The protein lysates of

615 HA-UB expressed in parental or LM05 cells were immunoprecipitated with an anti-NIK

616 antibody and then immunoblotted with an anti-HA antibody. B. Western blotting (upper) and

617 qRT-PCR (lower, $\mathrm{n}=3$, Welch's $\mathrm{t}$ test) analysis of cIAP1 expression in parental and LM05 cells.

618 C. Assessment of cIAP1 protein stability in parental and LM05 cells treated with $10 \mu \mathrm{g} / \mathrm{mL}$

619 cycloheximide (CHX). Quantification of cIAP1 protein level normalized by $\alpha$-Tubulin protein

620 level in each time point ( $\mathrm{n}=3$, two-way ANOVA followed by Bonferroni's multiple comparisons

621 test). D. Assessment of nascent cIAP1 protein levels in parental and LM05 cells using a click

622 reaction. Parental and LM05 cells were treated with $50 \mu \mathrm{M}$ L-homopropargyl glycine (HPG) for

$62324 \mathrm{hr}$. The nascent proteins labeled with HPG were conjugated to biotin using a click reaction.

624 The biotinylated proteins were purified with streptavidin beads and subjected to western blotting.

625 All data are representative of three independent experiments and are shown as the mean \pm SEM.

626 NS, not significant.

628 Figure 3. NIK upregulation in LM05 cells contributed to colony formation activity but not 629 cancer stemness.

630 A. Validation of NIK knockdown efficiency via Western blot (upper) and qRT-PCR (lower, n=3, 631 one-way ANOVA followed by Tukey's multiple comparison test) analyses in LM05-shGFP, 
shNIK no.1 and shNIK no.2 cells. For western blotting, all cell lines were either untreated or

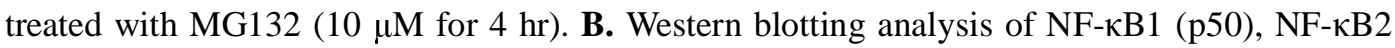
(p100/52), RelA and RelB in the nuclear extracts of LM05-shGFP, shNIK no.1 and shNIK no.2 cells. C. Cell growth curves of LM05-shGFP, shNIK no.1 and shNIK no.2 cells from planar culture ( $\mathrm{n}=3$, one-way ANOVA followed by Tukey's multiple comparison test). D. Representative images (upper) and quantification data (lower) ( $\mathrm{n}=5$, one-way ANOVA followed by Tukey's multiple comparison test) of the soft agar colony formation assay in LM05-shGFP, shNIK no. 1 and shNIK no. 2 cells. The scale bar is $5 \mathrm{~mm}$. E. Representative images (upper) and quantification data (lower) ( $\mathrm{n}=3$, one-way ANOVA followed by Tukey's multiple comparison test) from the flow cytometry analysis of the cancer stem cell populations in LM05-shGFP, shNIK no. 1 and shNIK no. 2 cells. F. Representative images (upper) and quantification data (lower) ( $\mathrm{n}=3$, one-way ANOVA followed by Tukey's multiple comparison test) of the mammosphere culture assay in LM05-shGFP, shNIK no.1 and shNIK no. 2 cells. All data are representative of three independent experiments and are shown as the mean \pm SEM. NS, not significant. $* \mathrm{P}<0.05$.

\section{Figure 4. NIK upregulation facilitated inherent tumorigenicity but not the lung metastatic} potential of LM05 cells.

A. Representative images of primary tumors (upper) and quantification data of the primary tumor weights (lower) ( $\mathrm{n}=4$, one-way ANOVA followed by Tukey's multiple comparison test) in LM05-shGFP, shNIK no. 1 and shNIK no. 2 cells. The scale bar is $1 \mathrm{~cm}$. B. Representative in vivo bioluminescent images of LM05-shGFP and shNIK no.2 cells (upper). Tumor growth curves (lower) (n=6 per group, two-way ANOVA followed by Tukey's multiple comparison test) of 
655 NOD-SCID mice orthotopically injected with LM05-shGFP, shNIK no.1 and shNIK no.2 cells.

656 C. Representative in vivo bioluminescent images (upper) and quantification data of the lung 657 metastasis tissue (lower) (one-way ANOVA followed by Tukey's multiple comparison test) 658 derived from NOD-SCID mice orthotopically injected with LM05-shGFP ( $\mathrm{n}=5$ ), shNIK no.1 659 ( $\mathrm{n}=4)$ and shNIK no.2 cells $(\mathrm{n}=5)$. D. Representative HE and IHC staining images (upper) and 660 quantification data (lower) (one-way ANOVA followed by Tukey's multiple comparison test) of 661 NIK protein production in lung metastasis tissue derived from NOD-SCID mice orthotopically 662 injected with LM05-shGFP, shNIK no.1 and shNIK no.2 cells. The average CAM5.2-positive 663 percentages were calculated from 5 fields of view from $n=4$ individual lung metastasis slides. 664 The scale bar is $500 \mu \mathrm{m}$. E. Representative HE and IHC staining images (upper) and 665 quantification data (lower) (one-way ANOVA followed by Tukey's multiple comparison test) 666 from the TUNEL assay and $\alpha$-SMA protein production in primary tumor tissues derived from 667 NOD-SCID mice orthotopically injected with LM05-shGFP, shNIK no.1 and shNIK no.2 cells. 668 The average number of TUNEL- and $\alpha$-SMA-positive cells were calculated from 5 fields of 669 view from $\mathrm{n}=4$ individual primary tumor slides. The scale bar is $500 \mu \mathrm{m}$. F. Representative 670 images (upper) and quantification data (lower) ( $\mathrm{n}=5$, one-way ANOVA followed by Tukey's 671 multiple comparison test) of the Boyden chamber assay with TIG-3 cells that were co-cultured 672 with LM05-shGFP, shNIK no.1, and shNIK no.2 cells. The scale bar is $500 \mu \mathrm{m}$. G. Western 673 blotting (upper) and immunofluorescence staining (lower) of $\alpha$-SMA expression in TIG-3 cells 674 that were co-cultured with LM05-shGFP, shNIK no.1 and shNIK no.2 cells or noncells (NC). 675 The scale bar is $50 \mu \mathrm{m}$. All data are shown as the mean \pm SEM. NS, not significant. ${ }^{*} \mathrm{P}<0.05$. 
678 A. Heatmap and Venn diagram of the DEGs from the LM05-shGFP, LM05-shNIK no.1 and 679 LM05-shNIK no.2 RNA-seq data, each performed in duplicate. Hierarchical analysis of the 680 heatmap was performed by the complete linkage method. Venn diagram of the signature genes 681 in LM05-shNIK cells. B. GSEA enrichment plot of the hallmark gene sets for the differentially 682 expressed genes in LM05-shNIK cells compared with LM05-shGFP cells. C. Validation of NIK 683 signature gene expression related to tumor inflammation using qRT-PCR (lower) (n=3, one-way 684 ANOVA followed by Tukey's multiple comparison test) in LM05-shGFP, shNIK no.1 and 685 shNIK no. 2 cells. All data are shown as the mean \pm SEM. NS, not significant. $* \mathrm{P}<0.05$.

Figure 6. NIK protein production increased in malignant breast cancer tissue.

688 A. Representative IHC staining images of NIK protein production in normal breast tissue and 689 breast tumors. The scale bar is $500 \mu \mathrm{m}$. B. Quantification data of the NIK IHC staining images 690 in normal breast tissue and breast tumors ( $(n=10$ normal, $n=6$ stage $I, n=72$ stage II, and $n=22$ 691 stage III); one-way ANOVA followed by Tukey's multiple comparison test). C. The graphical 692 summary indicates that the abnormal accumulation of NIK due to reduced translation of cIAP1 693 enhances tumorigenesis by promoting an inflammatory cancer microenvironment. All data are shown as the mean \pm SEM. NS, not significant. $* \mathrm{P}<0.05$. 
699 Western blotting analysis of NIK protein production in parental, LM05-Venus and

700 LM05-TAP-cIAP1 (murine) cells. For western blotting, all cell lines were either untreated or 701 treated with MG132 (10 $\mathrm{MM}$ for $4 \mathrm{hr})$. All data are representative of three independent 702 experiments.

Supplementary Figure 2. NIK ectopic expression partially rescued the reduction in tumorigenicity induced by NIK knockdown.

A. Validation of the NIK rescue efficiency via Western blot analysis in LM05-shGFP, shNIK no. 2 and NIK rescue cells. For western blotting, all cell lines were either untreated or treated

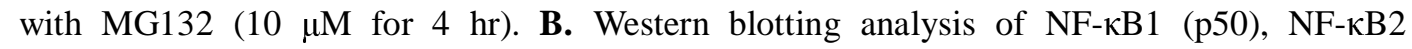
(p100/52), RelA and RelB in the nuclear extracts of LM05-shGFP, shNIK no.2 and NIK rescue cells. C. Tumor growth curves (two-way ANOVA followed by Tukey's multiple comparison test) of NOD-SCID mice orthotopically injected with LM05-shGFP ( $\mathrm{n}=6)$, shNIK no.2 ( $\mathrm{n}=6$ ) and NIK $(n=4)$ rescue cells. All data are representative of three independent experiments and are shown as the mean \pm SEM. NS, not significant. $* \mathrm{P}<0.05$.

Supplementary Figure 3. The upstream regulators of the downregulated common DEGs in NIK knockdown cells contained some genes associated with NF-кB signaling.

717 A. Ingenuity pathway analysis suggested statistically significant upstream regulators of the 718 downregulated common DEGs in NIK knockdown cells (selected activation z-score $<-2$ ). The 719 bar color indicates the molecule type of the upstream regulator (orange: cytokine, blue: 720 transcription regulator, yellow: kinase, green: receptor). The dotted line shows a p-value $=0.05$. 
722 Supplementary Figure 4. NIK expression was not correlated with PR, ER, or HER2 scores.

723 A. Quantification data of the NIK IHC staining images of normal breast tissue and breast tumors

724 for each PR score ( $\mathrm{n}=47$ - (negative), $\mathrm{n}=26+, \mathrm{n}=12++$, and $\mathrm{n}=24+++$; one-way ANOVA

725 followed by Tukey's multiple comparison test). B. Quantification data of the NIK IHC staining

726 images of normal breast tissue and breast tumors for each ER score ( $\mathrm{n}=35$ - (negative), $\mathrm{n}=17+$,

$727 \mathrm{n}=26++$, and $\mathrm{n}=41+++$; one-way ANOVA followed by Tukey's multiple comparison test). $\mathbf{C}$.

728 Quantification data of the NIK IHC staining images of normal breast tissue and breast tumor for

729 each HER2 score ( $\mathrm{n}=72$ - (negative), $\mathrm{n}=3+, \mathrm{n}=3++$, and $\mathrm{n}=31+++$; one-way ANOVA followed

730 by Tukey's multiple comparison test).

731

732 Supplementary Figure 5. The TVI model showed that the lung metastatic potential of

733 LM05 cells was not enhanced compared with parental cells.

734 A. Representative in vivo bioluminescent images (left) and quantification data of lung 735 metastases (right) (one-way ANOVA followed by Tukey's multiple comparison test) derived 736 from NOD-SCID mouse tail vein injection with parental, LM05, or LM1-2-1 cells ( $\mathrm{n}=5$ ).

738 Supplementary Figure 6. Some known lung metastasis-promoting genes are highly 739 expressed in the LM05 cell line independent of NIK knockdown.

740 A. Venn diagram of the upregulated genes in LM05 cells based on previous microarray 741 expression data and the downregulated genes found in the NIK KD cell line based on the 
bioRxiv preprint doi: https://doi.org/10.1101/2021.08.27.457878; this version posted August 28, 2021. The copyright holder for this preprint (which was not certified by peer review) is the author/funder. All rights reserved. No reuse allowed without permission.

742 RNA-seq data from this study. Expression of the known metastasis-promoting genes IL13RA2,

$743 T N S 1$, and EMP1 is independent of NIK knockdown. 
Fig.1

A

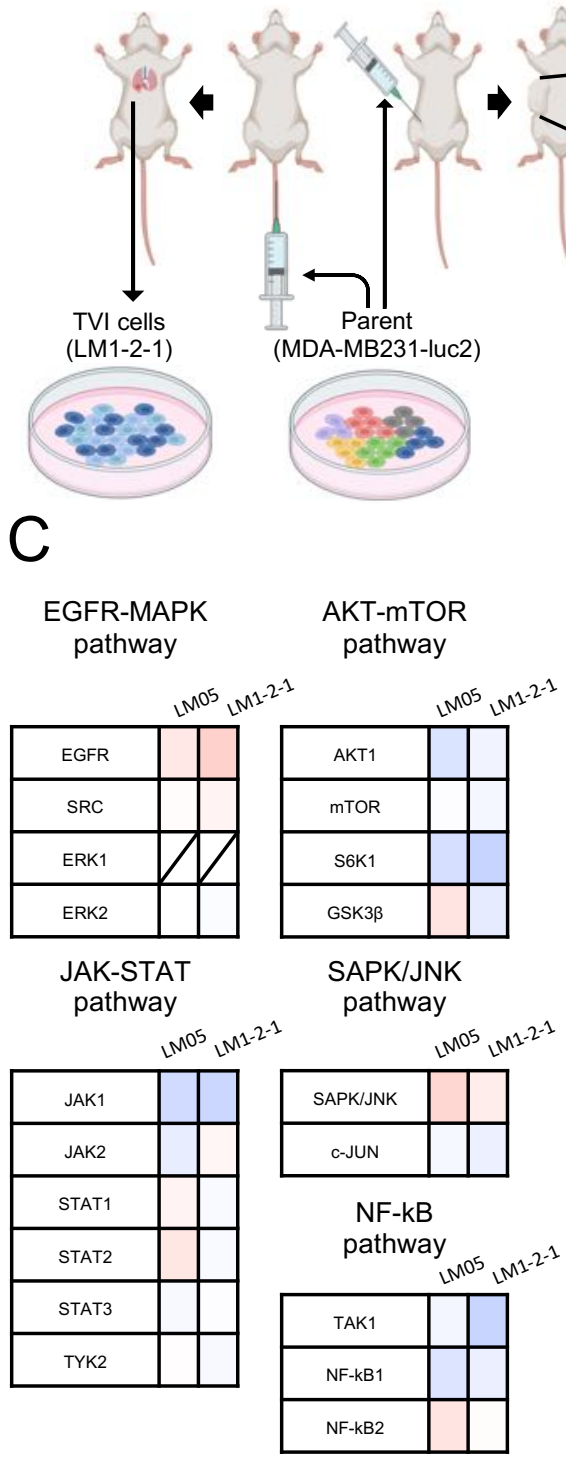

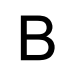
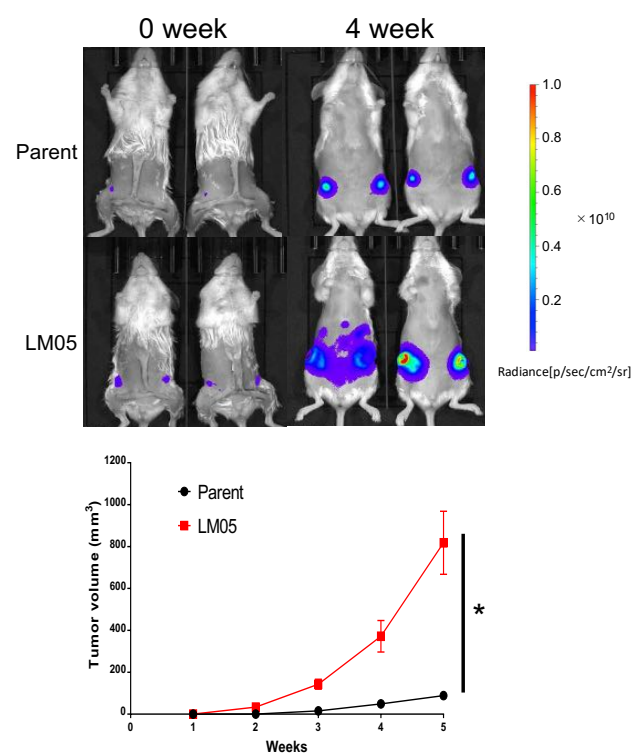

AKT-mTOR pathway
Cell adhesion pathway

D

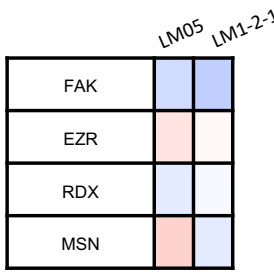

Other

pathways

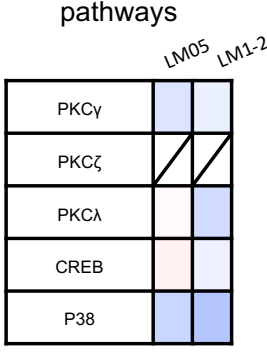

Microarray

expression index

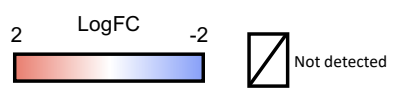

EGFR-MAPK pathway

LMO5 LMI-2-1

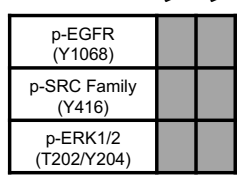

JAK-STAT

pathway

\begin{tabular}{|c|c|c|}
\hline $\begin{array}{c}\text { p-JAK1 } \\
(Y 1022,1023)\end{array}$ & & \\
\hline $\begin{array}{c}\text { p-JAK2 } \\
(Y 1007,1008)\end{array}$ & & \\
\hline p-SAT1(S727) & & \\
\hline p-SAT2(Y690) & & \\
\hline $\begin{array}{c}\text { p-SAT3(Y705) } \\
\text { p-TYK2 } \\
(Y 1054,1055)\end{array}$ & & \\
\hline
\end{tabular}

(Y1054,1055)

$\angle M 0^{5} \mathrm{LII}^{-2-1}$

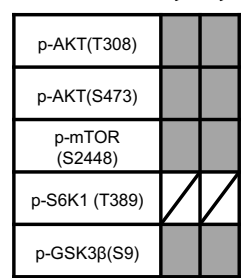

\section{SAPK/JNK pathway}

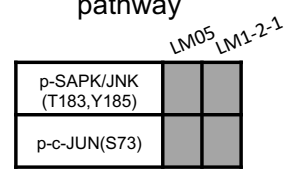

NF-kB

pathway

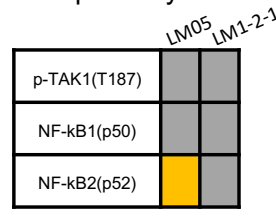

Cell adhesion pathway

$\angle M 0^{5} \mathrm{LNI}^{-2-1}$

\begin{tabular}{|c|l|l|}
\hline$p-F A K(Y 397)$ & & \\
\hline$p-F A K(Y 576,577)$ & & \\
\hline $\begin{array}{c}p-E R M \\
(T 567 / 564 / 558)\end{array}$ & & \\
\hline
\end{tabular}

Other

pathways

\begin{tabular}{|c|l|l|}
\hline $\begin{array}{c}\mathrm{p}-\mathrm{PKC} / / \lambda \\
(\mathrm{T} 410 / 403)\end{array}$ & & \\
\hline $\mathrm{p}-\mathrm{PKC} \mathrm{C}(\mathrm{T} 514)$ & & \\
\hline $\mathrm{p}-\mathrm{CREB}(\mathrm{S} 133)$ & & \\
\hline $\begin{array}{c}\mathrm{p}-\mathrm{P} 38 \\
(\mathrm{~T} 180, \mathrm{Y} 182)\end{array}$ & & \\
\hline
\end{tabular}

Western blotting expression index

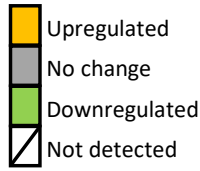

E

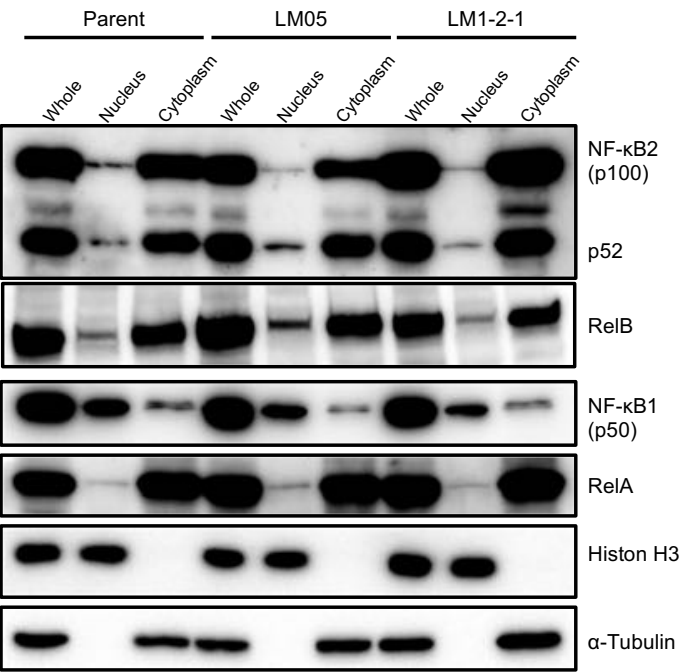

E
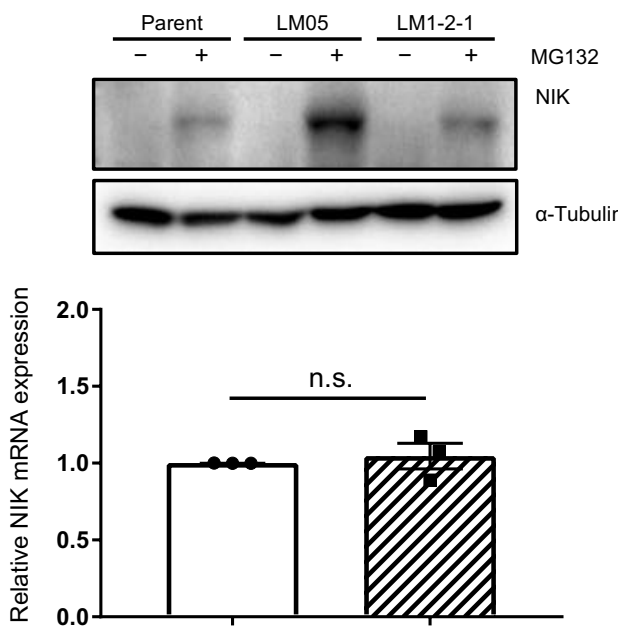
Fig.2

A

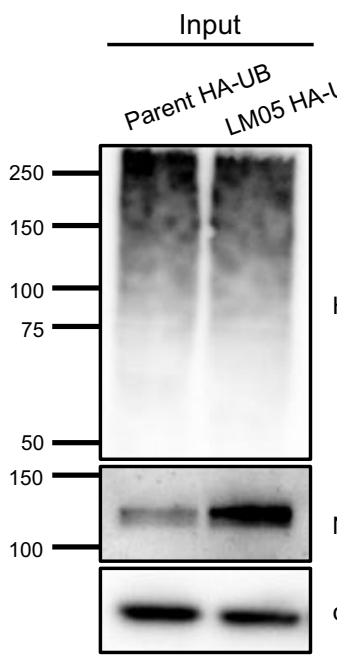

C
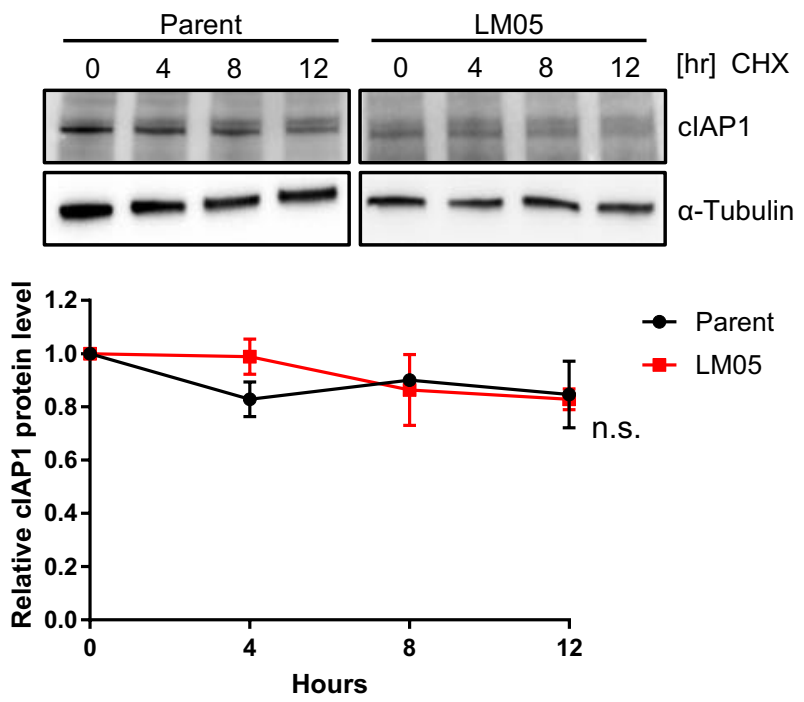

B

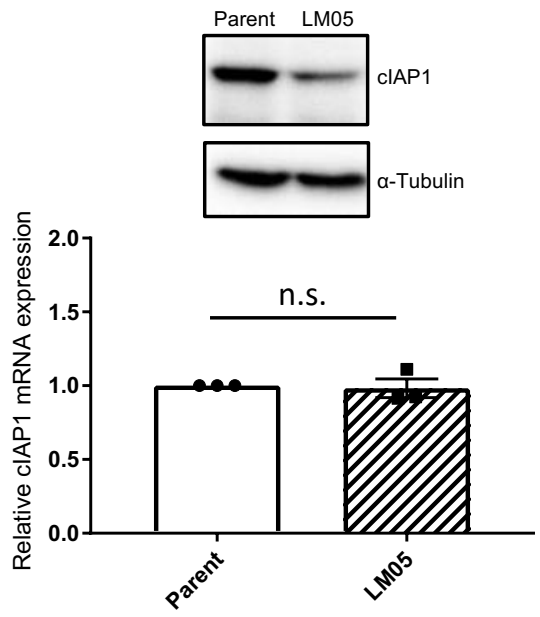

D Parent LM05 Parent LM05

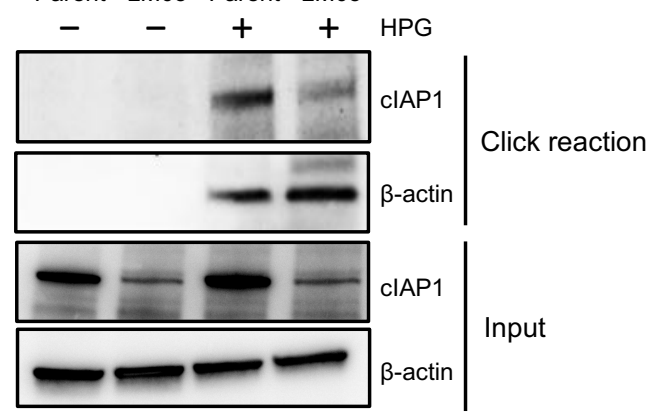


Fig.4

\section{A}

C

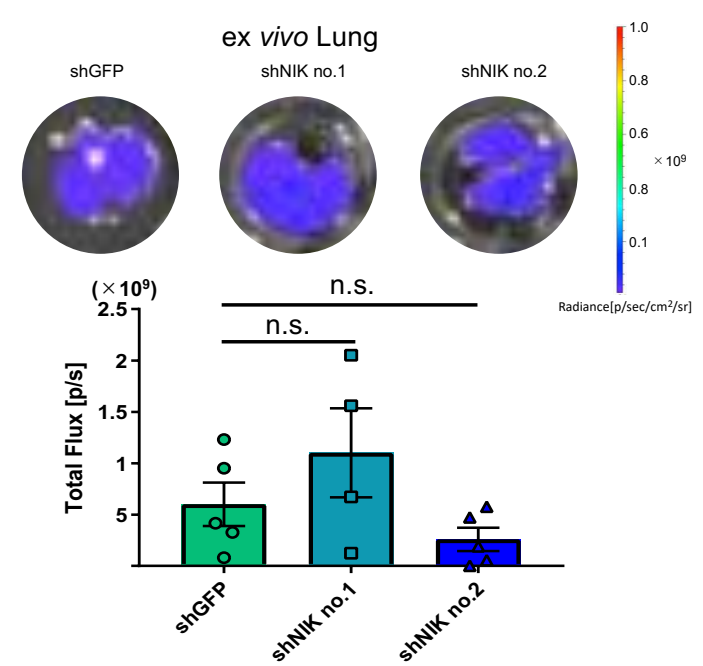

E

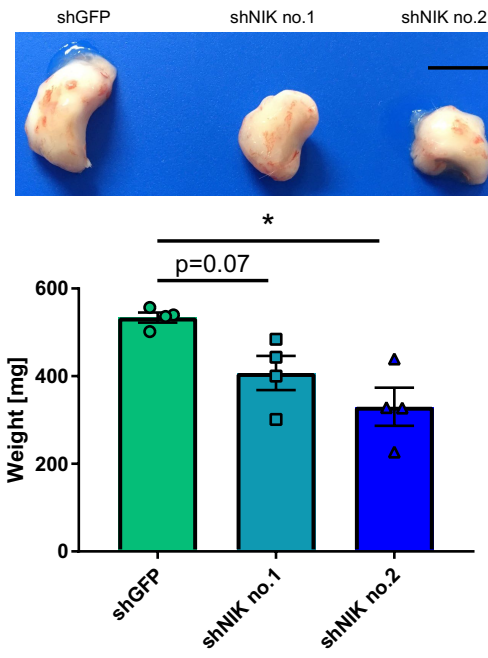

HE stain

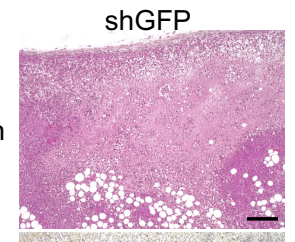

TUNEL
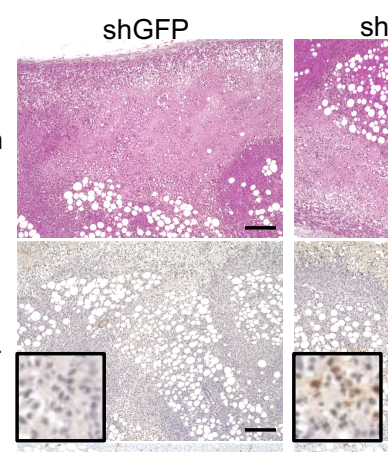

shNIK no.1
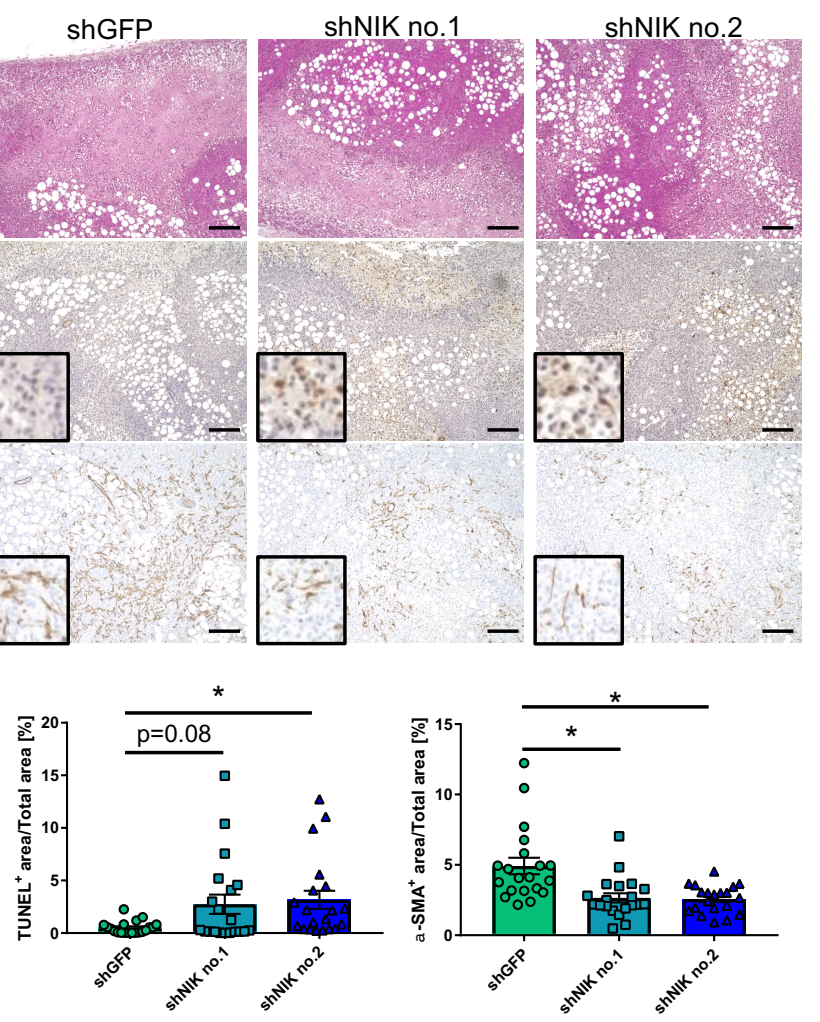
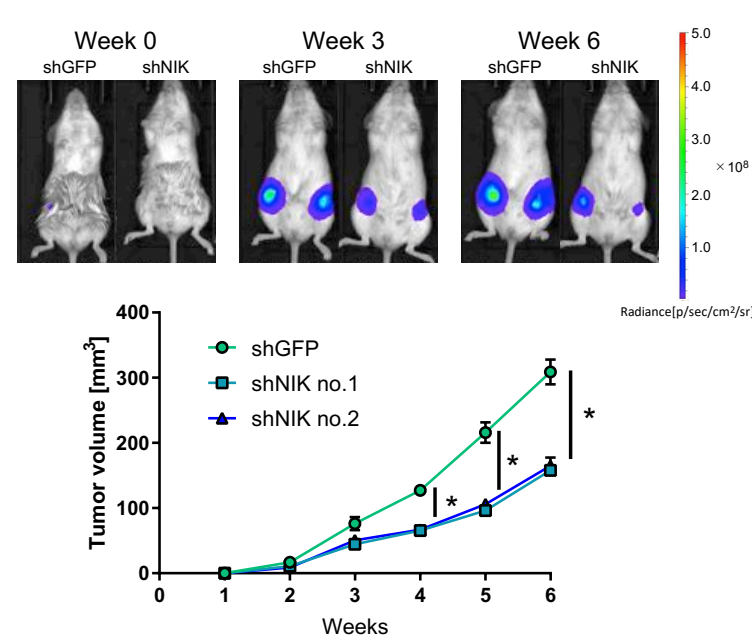

D

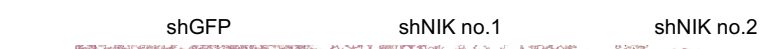

HE stain

F

ShGFP

shNIK no.1

shNIK no. 2

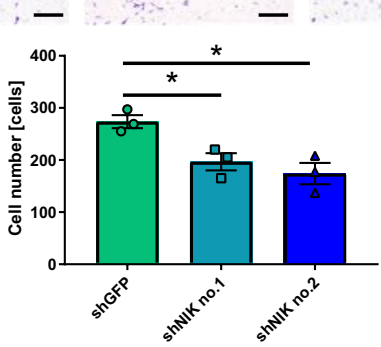

G
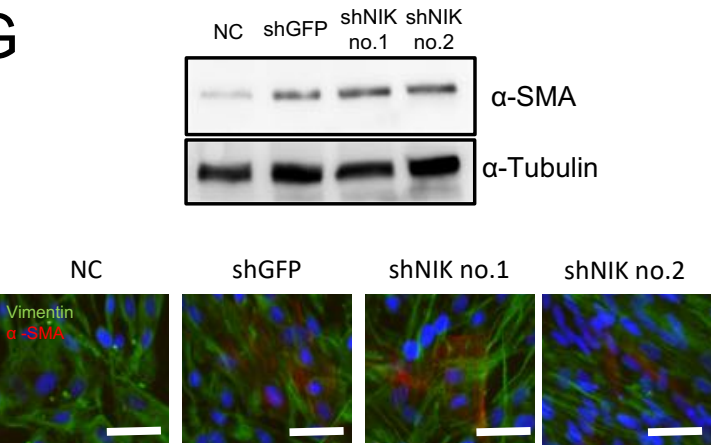
Fig.5

A

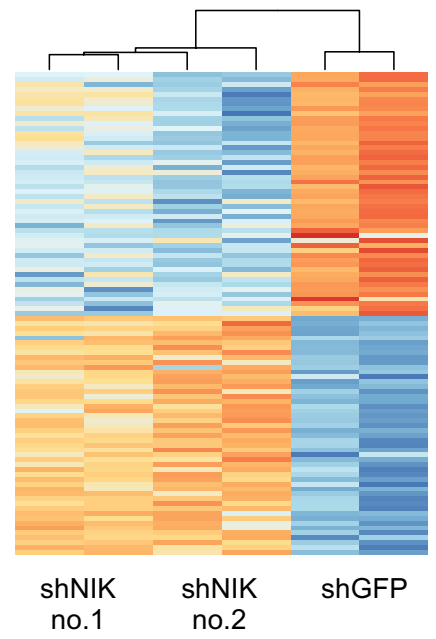

B

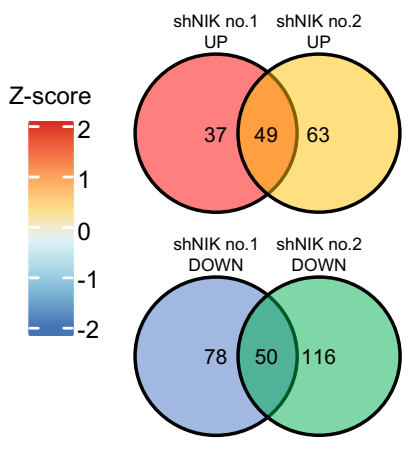

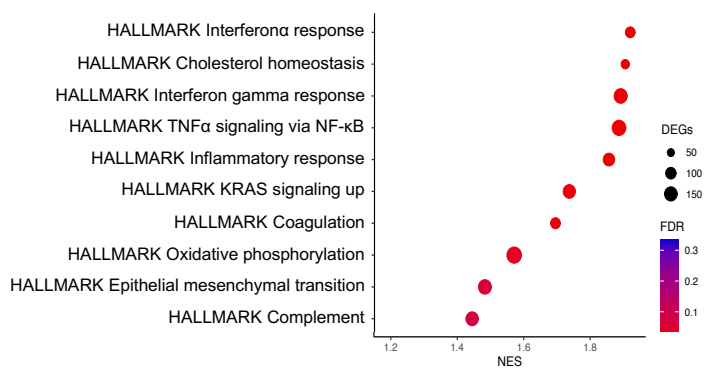

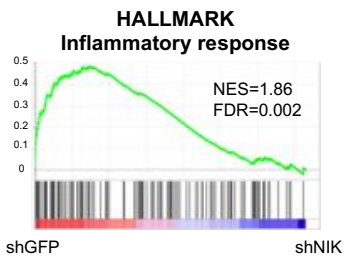

HALLMARK TNFa signaling via NF-KB

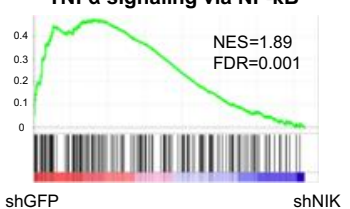

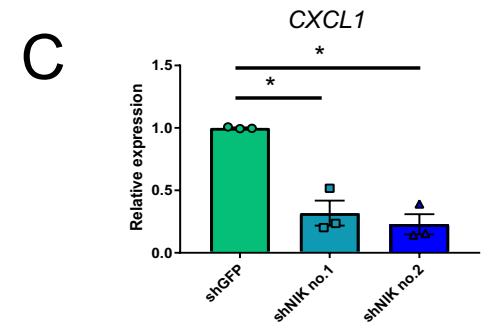
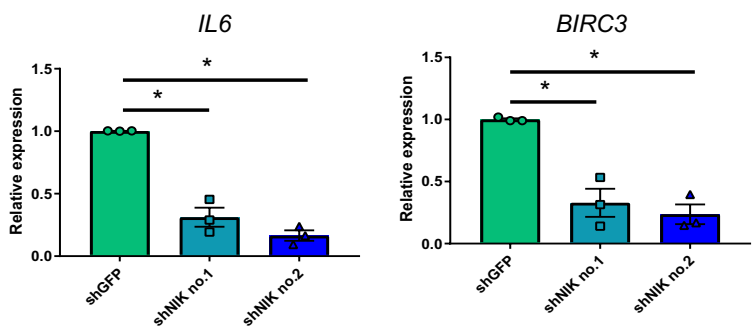
Fig.6

A
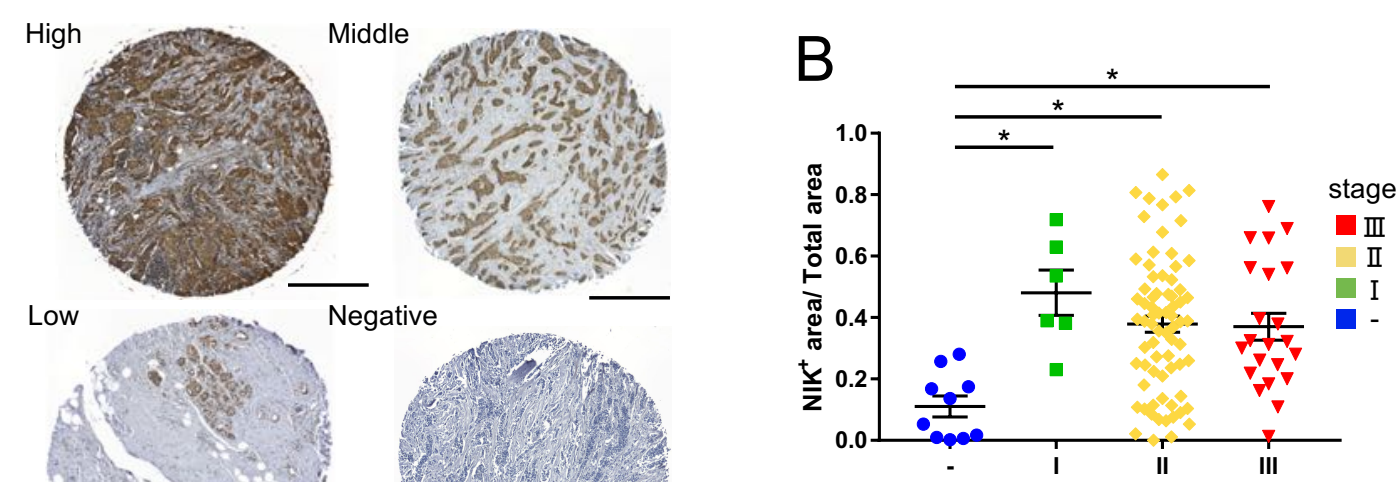

C

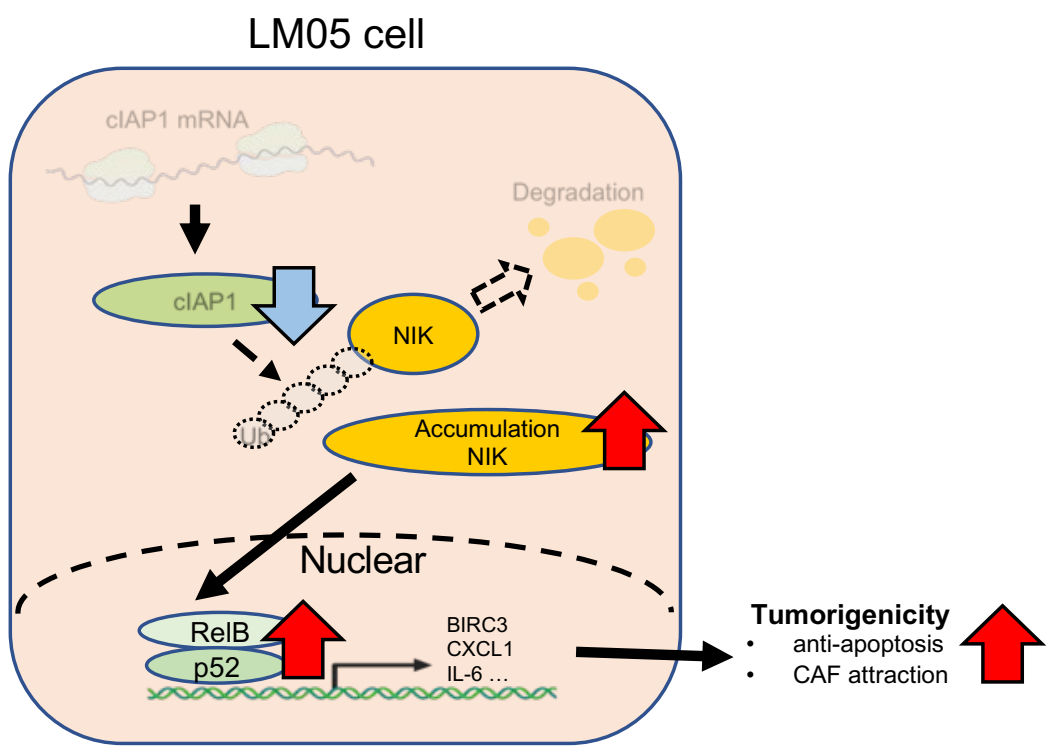


Fig.S2

A

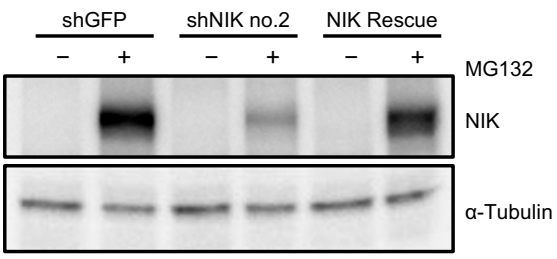

C

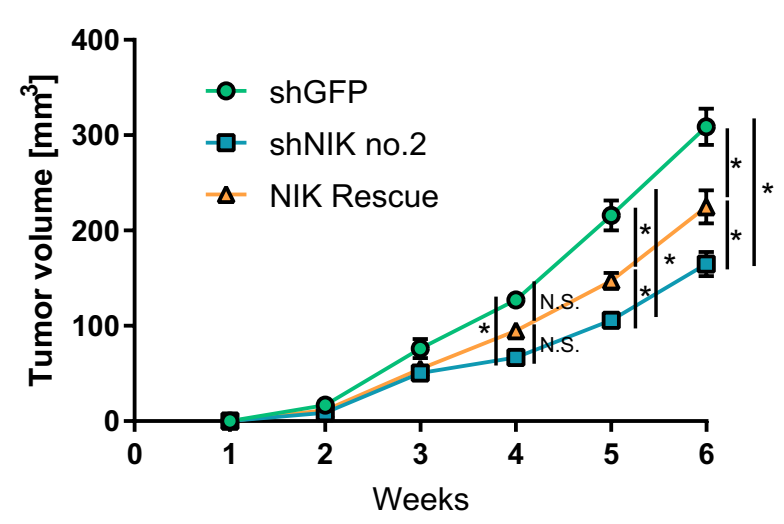

B

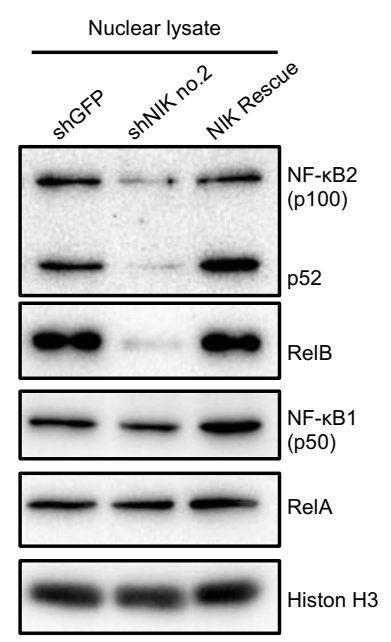

Weeks 
Fig.S3

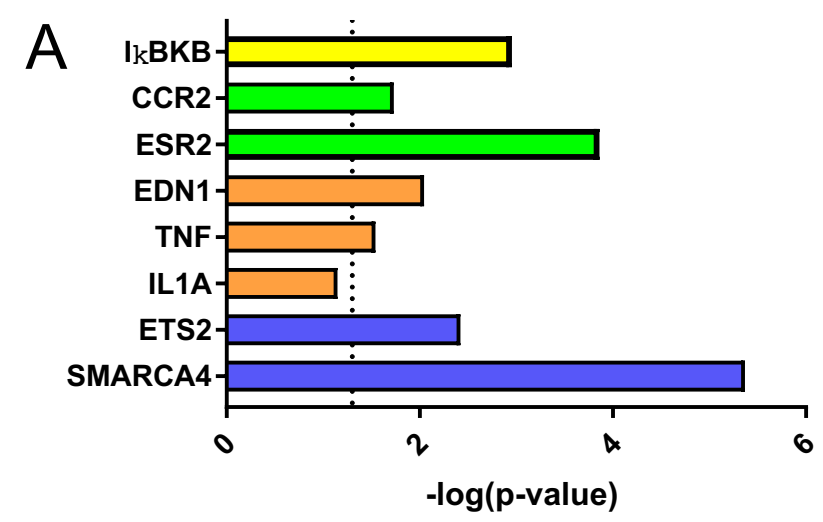


Fig.S4
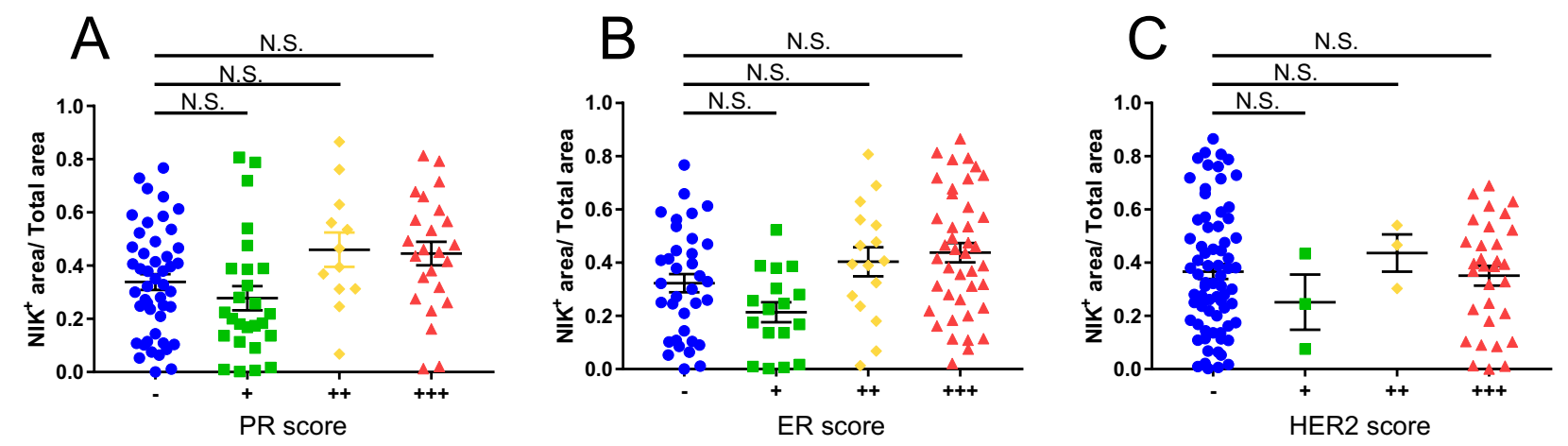
A

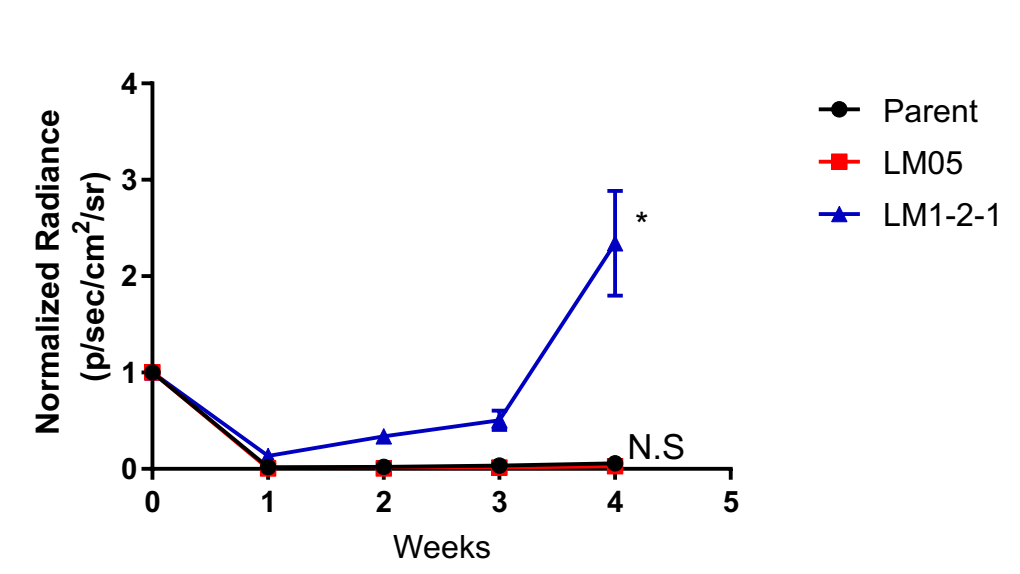

Fig.S5 
